\title{
Design quality assurance in cultural heritage restoration
}

\author{
Konstantin Tsvetkov* and Tatyana Volchenko \\ Moscow State University of Civil Engineering, Yaroslavskoe shosse, 26, Moscow,129337, Russia
}

\begin{abstract}
This study addressed the problem of quality assurance of scientific design documentation intended for cultural heritage restoration projects. The quality assurance systems, currently used by restoration companies in Russia, were considered. Such systems have disadvantages, implicated by the specific nature of restoration projects. It was proposed to introduce an additional quality assurance element, namely, appraisal of design solutions by a scientific methodological council. The main purpose and principles of the council's work were formulated. The proposal was tested by implementing such council in the quality assurance procedure of a Moscow restoration and design company in 2016-2018. The main criterion of the council's efficiency was the percentage of positive conclusions of the Historical-Cultural State Expert Review, obtained on the first try. By the end of the second year of the council's work, this indicator increased by $30 \%$ and approached $100 \%$. The finding of this research can be of interest to restoration companies and specialised organization that develop quality management systems.
\end{abstract}

\section{Introduction}

In Russia, preservation of cultural heritage is a licensable activity. Russian administrative law holds company executives responsible for violating licensing requirements. Moreover, according to Section 6 Article 45 of the Federal Law "On objects of cultural heritage (historical and cultural monuments) of the peoples of the Russian Federation" No. 73-FZ dated 25.06.2002, only natural persons, qualified by the federal authorities for cultural heritage protection, can be admitted to conservation and restoration of cultural assets that are listed in the Unified State Register of Cultural Heritage Objects or have been recently discovered. There are procedures for revoking the qualification of such specialists if in their professional activity they commit violations that result in damage to a cultural heritage object. These strict rules arise from the high cost of any mistake that can lead to an irreparable loss of cultural heritage of the peoples of Russia.

The conservator-restorer community have repeatedly discussed the need to improve the quality assurance (QA) mechanisms for restoration-related design processes. This study was conceived following one of such discussions at the II International Congress of Restorers in September 2015 in Kazan. We aimed to examine Russian experience in QA of

\footnotetext{
*Corresponding author: redfoxic@inbox.ru
} 
research and design documentation related to the cultural assets preservation, to analyse the weak points in the QAS, to develop improvement proposals, and test the improved QA methods in the work of a restoration company. This paper presents the main results of the examination and testing.

\section{Methods}

In an attempt to examine the domestic experience in QA of research and design documentation related to the cultural assets preservation, the following methods were selected:

- Questionnaire survey. Some multiple-choice questions were included to assess the presence/absence of a QAS, its content and efficiency.

The total number of design organizations licensed by the Ministry of Culture of Russia could be designated as a general population for the study. In this case, the total population would exceed 1500. However, we considered it permissible to significantly reduce this value by presenting the following requirements to the survey participants.

a. have a license to carry out activities related to the cultural assets preservation for at least 5 years before the start of the survey;

b. be a noticeable participant in the restoration market, having an annual revenue of $50 \mathrm{mln}$ rub or more and at least 5 state contracts signed per year;

c. be a specialized company that receives at least $75 \%$ of the total revenue from restoration of cultural heritage objects and restoration-related design activities.

As a result, the total population comprised about 90 organizations. The required sample size was calculated based on a confidence level of $90 \%$, a confidence interval of $5 \%$ and amounted to 68 organizations.

The questionnaires were sent to restoration 90 companies, and replies were received from the 71 recipients. The confidence interval was equal to $\pm 4.52 \%$.

Table 1 shows the geographical spread of the survey and the number of companies that returned the questionnaires.

Table 1. Geographical spread of the questionnaire survey

\begin{tabular}{|c|c|}
\hline Regions of the Russian Federation & Number of survey participants \\
\hline Moscow and Moscow Oblast & 26 \\
\hline Saint Petersburg and Leningrad Oblast & 22 \\
\hline Nizhny Novgorod Oblast & 7 \\
\hline Yaroslavl Oblast & 5 \\
\hline Sverdlovsk Oblast & 4 \\
\hline Republic of Tatarstan & 3 \\
\hline Kostroma Oblast & 2 \\
\hline Republic of Mordovia & 1 \\
\hline
\end{tabular}




\begin{tabular}{|c|c|}
\hline Bryansk Oblast & 1 \\
\hline Total: & 71 \\
\hline
\end{tabular}

Face-to-face interviews with executives of the restoration companies and officers of the governmental agencies for cultural heritage protection. The number of respondents was 42 people, incl. 31 participants, who were representatives of organizations that previously participated in the questionnaire survey. Considering the limitations of this kind of research, no quantitative assessment of the results was made.

In the interviews, we were able to assess the satisfaction of the company executives with the available QAS and to clarify the viewpoint of the governmental agencies on the existing QAS. Detailed notes were taken during each interview.

- Analysis of quotations to the state tenders, placed in the Unified Information System of Public Procurement.

We selected state tenders, in which the availability of QAS/QMS was a necessary criterion. The selected tenders were placed during the year preceding the start of this study. The quotations of the restoration companies were considered in addition to the information obtained from the questionnaires and the interviews.

- Selection and analysis of proposals from the companies that develop QAS/QMS.

We requested commercial proposals on the development and implementation of QAS for design documentation. The main condition was to take into account that the design documentation was intended for cultural heritage restoration projects. The purpose of this step was to assess the potential of QAS/QMS developers as it relates to the design processes for the purposes of cultural heritage restoration projects.

When analysing the obtained information, we sought to answer the following questions:

- if QAS are used by the restoration companies or not;

which risks associated with the design development a QAS can reduce or eliminate;

- and, most importantly, if the specific nature of restoration projects can be taken into account and embedded into the QAS?

The answers to these questions served to assess the efficiency of QAS applied by the restoration companies.

Further on, we determined the weak points in the QAS and proposed ways to mitigate them. The proposal was to introduce a Scientific Methodological Council as a QAS element. It was implemented into the working procedure of restoration and design company AK-Project, LLC (Moscow, Russia). Since the design solutions in the sphere of cultural asset restoration have to be submitted for the Historical-Cultural State Expert Review, the efficiency of using SMC in QA was evaluated by comparing the percentage of positive conclusions of the Historical-Cultural State Expert Review, obtained on the first try, before and after the introduction of SMC.

\section{Results and Discussion}

The study showed that the majority of restoration companies (83\% of respondents) applied QAS in the design development. At that, the QAS can basically be divided into 2 types.

Type I QAS were aimed mainly at checking the compliance of design documentation with the submission guidelines. In approximately $70 \%$ of cases, the controlled parameters included:

- completeness of documentation;

- correctness of execution and formatting;

- interconnection of sections; 
- correctness of references in the drawings;

- relevance of the explanatory (written) part to the graphic content.

About $30 \%$ of companies, having Type I QAS, reported that they evaluate only the compliance of design solutions per se to the current regulations.

It should be noted that companies that use Type I QAS, as a rule, did not engage a specialized organization to implement the QAS; most companies developed their QA procedures independently.

Type I QAS were usually organized in the following way: the QA check was performed at the level of the design department $(42 \%)$ or at the level of documentation release before delivery to the customer (34\%), and only in $24 \%$ of cases the design quality was monitored at both levels.

Type II QAS(30\% of respondents) were more complex, and the QA of project documentation was embedded in the general QMS that met the requirements of GOST R ISO 9001-2015 [1]. In these companies, the QMS was developed and certified by a specialized organization. It should be noted that the availability of QMS certification was an important criterion in the evaluation of quotations in the Unified Information System of Public Procurement. At the same time, the interviews with the company executives demonstrated low satisfaction with the available QMS. They were described as formal; keeping the QMS documents appeared cumbersome; designers distrusted this form of control; response to deviations from the quality standard and elimination of their causes were slow; and the QMS required large labour costs to ensure full functioning.

Thus, the following observations emerged from the analysis of the available QAS for design documentation in restoration companies:

- a majority of the restoration companies used QAS for design documentation in one form or another;

- the most controlled parameters were the compliance of the documentation with the requirements for execution/formatting and completeness; the interconnection of sections and compliance with the current regulations were checked less often;

- the QAS for design documentation, implemented as a part of the QMS, were regarded as inefficient by the company executives.

In addition to the above shortcomings, another serious disadvantage of the studied QAS was revealed. In accordance with GOST R 55528-2013[2], the design documentation intended for the preservation of cultural heritage objects, is categorized as scientific design documentation. The scientific component consists in the complex study of the cultural heritage object both at the stage preceding the design development and at the stage of repair and restoration works. The complex study includes historical, cultural, architectural, engineering, chemical-technological and other types of research, the results of which form the basis for the accepted restoration and design solutions. Simultaneously, the statutory regulation in terms of design solutions for restoration purposes is limited to several documents [2-5]. Hence the final quality of design solutions largely depends on the accuracy of the conducted research, the conformity of design solutions to the research results, and professional competencies of the designers. The available QAS for restorationrelateddesign documentation can only solve the task of assessing the compliance of the design solutions with the research results, while the accuracy of such assessment may require an additional study. All the other factors, arising from the specific nature of restoration projects, remain uncontrolled, and this is the principal weakness of the studied QAS. These findings confirm that there is an urgent need to improve the QA mechanisms for restoration-related design processes.

We requested commercial proposals from 12 companies that specialize in QAS/QMS development and asked for a QA solution that specifically addressed the above weaknesses. Seven proposals were received. However, the analysis of proposed solutions showed they 
were nearly the same as Type II QAS, considered above. Therefore, the problem requires principally new approach.

A possible solution can be to include an additional element or level of control in the Type I QAS. For this purpose, it appears reasonable to create a Scientific Methodological Council (SMC)affiliated with a professional organization of restorers oran association of restoration companies. A similar practice exists in some restoration organizations, for example, at State Unitary Enterprise "Central Scientific Restoration Design Workshops" (Moscow, Russia). Therefore, claiming no originality for the idea, this paper seeks to formulate the basic principles ofSMC organization and functioning, which make it possible to use it as a QA element for scientific design documentation.

The main objective of the SMCis to evaluate the quality of the adopted design solutions, based on the expert appraisal and followed by the recommendation to either approve the documentation for delivery to the customer, or to return for revision, or other. At that, the expert appraisal should not cover such formal aspects of as formatting, completeness, etc., which can remain under the responsibility of the regular QA procedures. The SMC should primarily evaluate the validity and essence of design solutions.

The purpose of SMCdetermines the main principles of its work:

1. The council should consist of the leading experts in the industry and qualified restorers of the first and the highest categories. At the same time, at least $30 \%$ of the council members should not have employment relations with the organization whose design documentation is being considered.

2. Any possibility of exerting undue influence on the council members should be eliminated, especially if such pressure comes from the management of the association that the council is affiliated to. For this, the following conditions should be met:

- when considering a design project, the council must include neither the heads of the company or department that developed the design project, nor the authors of the design project;

- the council's decision to return the design project for revision or correction cannot be re-negotiated or dismissed by the management of the restoration company that developed the design project;

- the financing of the council should not depend on the ratio of approved and returned projects.

3. It is advisable to divide the council into architectural and engineering sections.

4. The activities of the council should be regulated by a statute.

5. Council meetings should be held as soon as possible after a design project is submitted for consideration by the interested party. The necessity to appraise the design documentation should not delay the delivery of design documentation to the customer.

6. In order to support the interest of the professional community in the council's work, it may be recommended to include discussions of the current situation in the industry, preparation of legislative initiatives in the field of cultural assets conservation, etc. in the Council's agenda.

Having formulated these main principles, we also drafted the main documents regulating the council's work, i.e. the Statute, the MoM template, the template of SMC meeting notification and others.

In 2016-2018,the SMC was implemented intothe QAS of restoration and design company AK-Project, LLC. In the course of implementation, the following difficulties were successfully addressed:

Firstly, well-known experts, such as conservation architects of the highest category Mikhail B. Kanaev and Viktor F. Korshunov and conservation engineer of the highest category Natalia Yu. Tyutcheva, expressed interest in the council's work. The participation 
of such specialists immediately improved the credibility of the SMCand, hopefully, ensured the correctness of the council's decisions.

Second, the concerns of the designers that the only purpose of the council was to criticize their design, eventually disappeared. Once the documentation was revised following to the experts' comments, it was obvious that the design solutions became more accurate and scientifically grounded. The designers could use the experts' comments to improve their knowledge.

Third, at first the need to review and revise the design documentation led to late delivery of documentation to the customer, and the management of the restoration company had to deliver some urgent projects without appraisal by the council. However, as the positive impact of the new QA step became more and more obvious, the number of design projects submitted for the expert appraisal increased significantly.

To assess the council'sefficiency in the QAS, it was decided to choose the percentage of positive conclusions of the Historical-Cultural State Expert Review, obtained on the first try, as the criterion. After 3 years of the council's work in AK-Project, this indicator increased by more than $30 \%$ and approached $100 \%$. During the experimental period, the staff composition in AK-Project, LLC remain the same and the complexity of design problems did not vary significantly. Therefore, this proves the high efficiency of introducing the SMC into QA.

At the same time, we would like to draw attention to the fact that QAS, used in the restoration practice of the European Union, has mechanisms similar to SMC in addition to standard control procedures [6], when a group of experts is involved in quality assessment. In particular, the authors had a chance to familiarize themselves with the existence of this practice during a visit to the European exhibition of restoration, preservation of monuments and the renovation of historic buildings Denkmal (Germany, Leipzig, November 2016)

\section{Conclusion}

This study of the QAS for restoration-related design documentation revealed their disadvantages can be implicated by the inability to take into account the specific nature of the cultural assets restoration projects and hence assess the quality of design solutions. Specialized organizations that develop and implement QAS/QMS seem to be unable to offer solutions to these problems. Thus, it was suggested to introduce appraisal of the design documentation by the SMC as an element of the QAS. To test the idea, such council was implemented into the working procedures of one of the leading restoration and design companies. As a result, this element of the QAS was found efficient since the percentage of positive conclusions of the Historical-Cultural State Expert Review, obtained on the first try, significantly increased.

The present findings might be useful for the conservator-restorer community. We believe that councils may improve the QA in design development intended for the preservation of cultural heritage objects.

\section{References}

1. GOST R ISO 9001-2015 Quality management systems. Requirements.

2. GOST R 55528-2013 Composition and content of scientific and project documentation for the conservation of cultural heritage. Monuments of history and culture. General requirements. 
3. Federal Law "On the objects of cultural heritage (historical and cultural monuments) of the peoples of the Russian Federation" No. 73-FZ dated 25.06.2002.

4. GOST R 55567-2013 The order of the organization and conducting technical engineering studies on researches on objects of cultural heritage Monuments of history and culture. General requirements.

5. GOST R 56905-2016 Performance of measurement, engineering-geodetic works on objects of the cultural heritage. General requirements.

6. International Organization for standardizations (ISO): Specification for Representation Dates and Times in Information Interchange (ISO 8601:198/ES 28601), Geneva:1998 